\title{
Hemispheric asymmetries during processing of immoral stimuli
}

\section{Lora M. Cope 1,2, Jana Schaich Borg ${ }^{3,4}$, Carla L. Harenski' , Walter Sinnott-Armstrong ${ }^{5}$, Debra Lieberman ${ }^{6 *}$, Prashanth K. Nyalakanti' ${ }^{1}$ Vince D. Calhoun ${ }^{1,7,8}$ and Kent A. Kiehl ${ }^{1,2,8 *}$}

1 Mind Research Network, Albuquerque, NM, USA

2 Department of Psychology, University of New Mexico, Albuquerque, NM, USA

${ }^{3}$ Department of Psychiatry and Behavioral Science, Stanford University School of Medicine, Stanford, CA, USA

${ }^{4}$ Department of Neurobiology, Stanford University School of Medicine, Stanford, CA, USA

${ }^{5}$ Department of Philosophy, Duke University, Durham, NC, USA

${ }^{6}$ Department of Psychology, University of Miami, Coral Gables, FL, USA

7 Department of Electrical and Computer Engineering, University of New Mexico, Albuquerque, NM, USA

${ }^{8}$ Department of Neuroscience, University of New Mexico, Albuquerque, NM, USA

\section{Edited by:}

Ronald Yeo, University of New Mexico, USA

\section{Reviewed by:}

James J. Bonaiuto, University of

Southern California, USA

Andrew C. Gallup, Princeton University,

USA

Joan C. Borod, Queens College and

The Graduate Center of the City

University of New York, USA

\section{${ }^{*}$ Correspondence:}

Debra Lieberman, Department of

Psychology, University of Miami, PO

Box 248185, Coral Gables, FL

33124-0751, USA.

e-mail:debra@miami.edu;

Kent A. Kiehl, Mind Research Network,

1101 Yale Boulevard NE, Albuquerque,

NM 87106, USA.

e-mail:kkiehl@unm.edu
Evolutionary approaches to dissecting our psychological architecture underscore the importance of both function and structure. Here we focus on both the function and structure of our neural circuitry and report a functional bilateral asymmetry associated with the processing of immoral stimuli. Many processes in the human brain are associated with functional specialization unique to one hemisphere. With respect to emotions, most research points to right-hemispheric lateralization. Here we provide evidence that not all emotional stimuli share right-hemispheric lateralization. Across three studies employing different paradigms, the processing of negative morally laden stimuli was found to be highly left-lateralized. Regions of engagement common to the three studies include the left medial prefrontal cortex, left temporoparietal junction, and left posterior cingulate. These data support the hypothesis that processing of immoral stimuli preferentially engages left hemispheric processes and sheds light on our evolved neural architecture.

Keywords: fMRI, functional laterality, morality, emotions, left cerebral hemisphere

\section{INTRODUCTION}

The lateralization of brain function is a consistent finding in neuroscience. The idea that the cerebral hemispheres are not functionally or structurally identical was noted over 130 years ago by Broca (1887), who described the human brain as being the "most asymmetrical" brain of all animals. It is well-known that functions such as language and motor control tend to be lateralized (Vallortigara et al., 1999), and recent research has shown that aspects of moral and emotional processing may be lateralized as well (Ross, 1984; Meadows and Kaplan, 1994; Borod et al., 1997; Gainotti, 1997, 2000; Demaree et al., 2005; Miller et al., 2010; Young et al., 2010). To the extent that neural circuitry reveals additional hypotheses regarding evolved function, the laterality of moral processing can shed light on additional facets of the neural and evolutionary underpinnings of social behavior.

Emotions - including arousal and valence - are integral aspects of moral processing (Greene and Haidt, 2002; Tangney et al., 2007; Young and Koenigs, 2007; Young and Saxe, 2009). They guide our (frequently intuitive) judgments about emotionally aversive harms, for instance. Results of studies examining hemispheric laterality of emotional processes have led to two working hypotheses: the right hemisphere hypothesis and the valence hypothesis (Demaree et al., 2005). The right hemisphere hypothesis posits that emotion processing is generally lateralized to the right hemisphere (Gainotti, 1997, 2000). Emotion communication (Wittling and Roschmann, 1993; Wittling, 1995), autonomic functions of emotion (Canli et al., 1998; Davidson, 1993), and the subjective experience of emotions (Davidson, 1998) are all thought to be preferentially processed in the right hemisphere. In contrast, the valence hypothesis claims that the right hemisphere is specialized for processing negative emotions whereas the left hemisphere is specialized for positive emotions (Ross, 1984; Meadows and Kaplan, 1994; Borod et al., 1997). Yet contrary to both lateralization hypotheses, results from other studies have failed to find hemispheric specialization for emotional processing (Braun et al., 2005; Tamietto et al., 2007).

One possible explanation for the mixed findings in the literature is that the lateralization of emotion has been studied primarily at a broad level, for example, comparing positive and negative emotion. A more narrowed focus on the functional domain of an emotion might lead to more nuanced patterns of how emotion is processed in the brain. For instance, the results of one study suggested that emotions related to social experiences may be more left-lateralized than those that are not (Ross et al., 1994). In Ross et al. (1994), subjects were injected with sodium amobarbital into the right and left carotid arteries for neurosurgical purposes, and were asked to recount verbally an emotional life event before and 
after the injection. Following right-sided inactivation, subjects' emotional stories were factually the same as before the injection, but they contained significantly more social content. According to the authors, when only left hemisphere functionality was intact, subjects retrieved "social emotions" associated with the memory to the exclusion of "basic emotions." Since this study, however, the lateralization of social emotion and cognition has not received much attention, a surprising fact given the recent burgeoning of social cognitive neuroscience research.

Here we focus on moral processing and assess the extent to which neural circuitry underlying moral judgments shows hemispheric lateralization. Given that the propensity for moral reasoning across multiple domains (e.g., incest, theft, physical assault, cheating, drug use, etc.) reflects the operation of evolved adaptations (e.g., Lieberman et al., 2003; Kurzban et al., 2010), we aim to identify its associated neural organization. Research on moral judgment has led to two-process theories, with implicit, automatic processes being driven by emotional responses, and explicit, controlled processes relying on cognitive control and reasoning (e.g., theory of mind, belief attribution, etc.; Haidt, 2001; Greene et al., 2004; Cushman et al., 2006). Functional imaging studies and studies of patients with brain damage suggest that there is a network of regions associated with the processing of moral stimuli (Greene and Haidt, 2002; Moll et al., 2005), which typically includes the medial prefrontal cortex, areas around the temporoparietal junction, and posterior cingulate, but can also include areas of the anterior cingulate, anterior temporal lobe, and other substantial portions of the limbic system. Despite an increase in our understanding of the specific brain regions that are involved in the processing of moral stimuli, the hemispheric laterality of this function has not been well-studied. Recent studies of one aspect of moral processing, belief attribution (Miller et al., 2010; Young et al., 2007, 2010; Young and Saxe, 2008, 2009), have implicated the right temporoparietal junction, though the medial prefrontal cortex, precuneus, and left temporoparietal junction have also been identified. Thus it is still unclear whether one hemisphere is more important for moral processing from the analyses carried out in these previous imaging studies.

Here we reanalyze data from three separate studies and test the hypothesis that processing of moral violations, which involves social emotion, is associated with functional left hemispheric asymmetry. Here we use the term "immoral" to represent a moral violation, "pro-moral" to represent a morally positive attitude or behavior, "moral" to represent stimuli with moral content (incorporating both "immoral" and "pro-moral"), and "nonmoral" to represent stimuli without moral content. Each of the three studies employed different methods to investigate moral psychology. Study 1 used a memory-recognition task where subjects were presented with four types of statements: pathogen-related, sexual immoral, non-sexual immoral, and neutral statements. This study looked at implicit moral processing, that is, the processing of moral stimuli without the explicit instruction to attend to moral content and make moral judgments (Schaich Borg et al., 2008). Study 2 required participants to explicitly judge as wrong or not wrong various non-controversial immoral, noncontroversial pro-moral, and morally controversial acts. In study 3, participants were asked to make explicit moral judgments of pictures with moral content ("immoral"), negative pictures without moral content ("non-moral"), and neutral pictures (Harenski et al., 2008).

By drawing upon these three diverse studies, we can examine the lateralization of moral processing across several paradigms utilizing both linguistic and pictorial stimuli, implicit and explicit processing, and different classes of moral stimuli (sexual and nonsexual). Given the left-lateralization of social emotional processing (Ross et al., 1994) and the dearth of direct assessment of the laterality of moral processing, we hypothesized that the processing of immoral stimuli, relative to non-moral stimuli, would also be left-lateralized.

\section{MATERIALS AND METHODS}

All participants were healthy, right-handed adults. Written, informed, institutional review board-approved consent was obtained from each participant.

\section{STUDY 1: IMPLICIT EVALUATIONS OF MORAL AND NON-MORAL ACTS IN A MEMORY-RECOGNITION TASK Participants}

Fifty males [mean age, 25 years; standard deviation $(S D)=6$ ] were recruited from Hartford Hospital and Yale University.

\section{Stimuli and task}

During scanning, participants were given a simple memoryrecognition task that has been shown previously to tap affective processing (Kiehl et al., 2005). Participants saw a total of four types of statements: statements involving pathogen-related acts (Pathogen; e.g., You eating your sister's spoiled hamburger, You sipping your sister's urine, You eating your sister's scab), incestuous acts (Incest; e.g., You giving your sister an orgasm, You watching your sister masturbate, You fondling your sister's nipples), nonsexual immoral acts (Non-Sexual Immoral; e.g., You burglarizing your sister's home, You killing your sister's child, You knocking your sister down the stairs), and neutral acts (Neutral; e.g., You reading to your sister, You holding your sister's groceries, You closing the door to your sister's closet). Statements were divided into short memorize-recognize blocks, where each block contained only one type of statement. During the memorize phase of each block, participants were presented with four statements to memorize. During the recognize phase, they were again presented with four statements, but here they were instructed to report whether each statement was from the previous memorize phase or a new statement. Two randomly selected statements from the memorize phase were shown in the recognize phase. A total of six statements were shown in each memorize-recognize block: two shown only in the memorize phase, two shown only in the recognize phase, and two shown in both the memorize and recognize phase.

Each statement was shown for $2500 \mathrm{~ms}$ with an intertrial interval of $500 \mathrm{~ms}$. Participants pressed one button if the statement was from the previous memorize phase or another button if it was a new statement. Participants completed two runs of 12 memorize-recognize blocks for a total of 144 statements (36 for each category of statement). After scanning, participants completed a questionnaire that asked how disgusting, how morally wrong, and how appealing each act was. $t$-Tests revealed that 
incest and non-sexual immoral acts were rated as equally immoral $(p=0.15)$, incest and pathogen stimuli were equally disgusting $(p=0.12)$, and incest and non-sexual immoral acts were equally unappealing $(p=0.51)$.

\section{MRI data acquisition and preprocessing}

See Table 1 for details on image acquisition and preprocessing. One contrast of interest was specified to evaluate the effects of the immoral acts (Incest + Non-Sexual Immoral; Immoral > Neutral) and another contrast of interest was specified to control for the effects of disgust processing (Immoral $>$ Pathogen). Incest $>$ Neutral and Non-Sexual Immoral $>$ Neutral were also evaluated separately to ensure that the effects of the Immoral $>$ Neutral contrast were not due solely to the sexual stimuli of the Incest condition.

\section{STUDY 2: EXPLICIT EVALUATION OF MORAL ACTS: JUDGING ACTS TO BE "WRONG" OR “NOT WRONG" \\ Participants}

Twenty-three individuals (mean age, 32 years; SD = 11) were recruited (15 female) from the Olin Neuropsychiatry Research Center in Hartford, CT, USA and Yale University.

\section{Stimuli and task}

During scanning, participants were shown three types of statements: 50 statements describing acts that most people think are immoral (e.g., drunk driving, stealing money, lying), 50 statements that most people think are pro-moral (e.g., working hard, feeding the hungry, being faithful), and 50 statements that are morally controversial (e.g., same-sex marriage, abortion, euthanasia). Participants pressed one button if they judged the act to be "wrong" and another button if they judged the act to be "not wrong." Participants were allowed a

Table 1 | MRI data acquisition and preprocessing.

\begin{tabular}{|c|c|c|c|}
\hline & Study 1 & Study 2 & Study 3 \\
\hline \multirow[t]{2}{*}{ Scanner } & Siemens & Siemens & Siemens \\
\hline & Allegra 3T & Allegra 3T & Allegra 3T \\
\hline Sequence & $\begin{array}{l}\text { Gradient } \\
\text { echo planar }\end{array}$ & $\begin{array}{l}\text { Gradient } \\
\text { echo planar }\end{array}$ & $\begin{array}{l}\text { Gradient } \\
\text { echo planar }\end{array}$ \\
\hline $\begin{array}{l}\text { Repetition } \\
\text { time (TR, ms) }\end{array}$ & 1500 & 1500 & 1500 \\
\hline Echo time (TE, ms) & 27 & 27 & 27 \\
\hline Flip angle & $70^{\circ}$ & $70^{\circ}$ & $65^{\circ}$ \\
\hline $\begin{array}{l}\text { Field of view } \\
\text { (FOV, cm) }\end{array}$ & 24 & 24 & 24 \\
\hline Acquisition matrix & $64 \times 64$ & $64 \times 64$ & $64 \times 64$ \\
\hline Voxel size (mm) & $3.75 \times 3.75 \times 4$ & $3.75 \times 3.75 \times 4$ & $3.44 \times 3.44 \times 5$ \\
\hline Gap/slices & $1 \mathrm{~mm} / 29$ & $1 \mathrm{~mm} / 29$ & $0 \mathrm{~mm} / 30$ \\
\hline $\begin{array}{l}\text { Spatial smoothing } \\
\text { (FWHM) }\end{array}$ & 12 & 9 & 8 \\
\hline Software & SPM5 & SPM5 & SPM5 \\
\hline
\end{tabular}

After data acquisition, each run was realigned to the first scan of the run using INRlalign (Freire et al., 2002). They were then motion corrected, normalized to a standard MNI template, and spatially smoothed. Low frequency noise was removed using a high-pass filter. maximum of $10 \mathrm{~s}$ to respond, and responses were followed by $1-6 \mathrm{~s}$ of black screen. The next stimulus began immediately. Stimuli were randomized and counter-balanced over three runs.

\section{MRI data acquisition and preprocessing}

See Table 1 for details on image acquisition and preprocessing. One contrast of interest was specified to evaluate the brain regions selectively involved in judging controversial acts to be wrong (i.e., requiring substantial moral processing) vs. judging non-controversial acts to be wrong (i.e., requiring less moral processing): Controversial Wrong > Non-Controversial Wrong.

\section{STUDY 3: EXPLICIT EVALUATION OF PICTURES DEPICTING MORAL ACTS: JUDGING HOW WRONG IS EACH ACT \\ Participants}

Thirty-one individuals were recruited from the Olin Neuropsychiatry Research Center and Trinity College in Hartford, CT, USA. One participant was excluded for excessive head motion during scanning, one was excluded for poor task performance (the participant missed several ratings across both runs), one was excluded for psychiatric medication, and one was excluded for experimenter error, leaving 27 participants (mean age, 25 years; $\mathrm{SD}=4 ; 13$ female).

\section{Stimuli}

During scanning, participants were shown three types of pictures: 25 immoral, 25 non-moral, and 25 neutral. Pictures were taken from the International Affective Picture System (IAPS; Lang et al., 1995), and supplemented with pictures from the popular media. Immoral pictures depicted negatively valenced scenes with clear moral content (e.g., an individual being assaulted, a hand breaking into a house, a person drinking and driving). Non-moral pictures depicted negatively valenced scenes without moral content (e.g., two individuals arguing, a mutilated hand, a person exhibiting "road rage"). Neutral pictures were neither positively nor negatively valenced and contained no moral content (e.g., a conversation, a hand being fingerprinted, a person driving normally). Pictures categorized as immoral and non-moral were selected as such based on moral content ratings of a larger sample of pictures by two separate groups of participants (Harenski and Hamann, 2006). Immoral and non-moral pictures were matched on emotional arousal and social complexity (Harenski and Hamann, 2006; Harenski et al., 2008).

\section{Task}

Participants were instructed that they would see a series of pictures depicting people and events. For each picture, participants were told to decide whether it contained a moral violation. If they judged the picture to contain a moral violation, they were told to rate the severity of the violation on a scale from 1 (no violation) to 5 (most severe violation). If they judged the picture to contain no moral violation, they were instructed to give a rating of 1 . It was emphasized that participants should make judgments based on their own opinions, not what society or other people would think.

Each picture was displayed for $6 \mathrm{~s}$. After $6 \mathrm{~s}$, a rating scale was displayed, which consisted of a red bar that grew continuously from 1 to 5 over the course of $4 \mathrm{~s}$. Participants pressed a button to stop the bar when it reached the number corresponding to their 
desired rating of that particular picture. Immoral, non-moral, and neutral pictures were presented in randomized order, and 25 null events of the same duration as picture trials consisting of a black screen and white fixation cross were randomly interspersed with the pictures, creating jitter between picture trials. The 100 total trials (immoral, non-moral, neutral, and fixation) were divided into two equal runs with 50 trials occurring in each run.

\section{MRI data acquisition and preprocessing}

See Table 1 for details on image acquisition and preprocessing. Two contrasts of interest were specified: one that controlled for the effects of general emotional processing (Immoral > Non-Moral) and a control contrast that evaluated the effects of no moral violation (Non-Moral > Neutral).

\section{FUNCTIONAL HEMISPHERIC ASYMMETRY STATISTICAL ANALYSIS}

The hemispheric asymmetry statistical procedure developed by our group is described in detail elsewhere (Stevens et al., 2005). After completing the first-order statistics for each study, the data were spatially normalized to a hemispherically symmetrical template in order to directly compare hemodynamic activity between the left and right hemispheres. A symmetrical template was needed because of the anatomical differences between the two hemispheres that exist in the default Montreal Neurological Institute (MNI) template provided with SPM. The symmetrical template was created by flipping the SPM5 echo planar imaging (EPI) template along the $y$-axis to create two images: one with the left hemisphere on both sides and one with the right hemisphere on both sides. These images were then averaged to create the symmetrical template. We then calculated the parameters necessary to spatially normalize each participant's images to the symmetrical template, thereby providing a less anatomically biased way of comparing hemodynamic activity in each hemisphere.

After these parameters were calculated, we compared hemodynamic activity in left hemisphere voxels and right hemisphere voxels in order to identify areas where the amplitude of the hemodynamic response differed between the two hemispheres. For each voxel in the right hemisphere, the amplitude of the hemodynamic response was subtracted from the corresponding voxel in the left hemisphere and vice versa in order to produce images with positive voxels in both hemispheres. The images for each participant and each condition of interest were then used in a series of secondlevel random effects SPM analyses. We performed one-sample $t$-tests on the contrast images for each task's contrasts of interest. Significant peak voxel coordinates and $t$-score statistics are reported for local maxima.

After processing, additional steps were taken in order to identify asymmetry effects. For each positive voxel, there could be one of three types of relationships: (a) positive signal change in one hemisphere and negative signal change in the other, (b) positive signal change in one hemisphere that is greater than the positive signal change in the other, and (c) negative signal change in one hemisphere that is smaller than negative signal change in the other. In order to address this issue, masks were created for each type of relationship (positive vs. negative, positive vs. positive, and negative vs. negative) and were applied to the images from the asymmetry analyses. These relationships are presented as different colors in the figures: red $=$ both sides positive; blue = left positive, right negative; yellow = left negative, right positive; green $=$ both sides negative. There were no significant left negative, right negative (i.e., green) voxels. Finally, a step was needed to account for the possibility of finding a significant laterality effect where no significant main effect of condition existed. Therefore, main effects masks for each contrast of interest were created and applied to the data from the functional asymmetry analyses.

\section{CONJUNCTION ANALYSIS}

Another potential method for assessing the laterality and commonality among three studies is to perform a conjunction analysis on each contrast of interest. The conjunction analysis is a stringent test, and evaluates regions that are common to all three studies (i.e., a logical AND statement) for the specific contrasts of interest (Nichols et al., 2005). A conjunction analysis was performed using SPM5 defaults that incorporated Study 1 (Immoral > Pathogen), Study 2 (Controversial Wrong > Non-Controversial Wrong), and Study 3 (Immoral > Non-Moral). A subtraction procedure was then used to lateralize the images by determining the hemisphere with the higher $t$-value for each cluster.

\section{RESULTS}

Functional magnetic resonance imaging (fMRI) was utilized in three studies investigating the neural correlates of moral processing (Harenski et al., 2008; Schaich Borg et al., 2008). Laterality analyses were then performed (Stevens et al., 2005; Figure 1; Table 2).

\section{STUDY 1}

The main effects of non-sexual immoral, incest, and pathogen statement processing have been described elsewhere (Schaich Borg et al., 2008), and the main effects of Immoral $>$ Neutral and Immoral $>$ Pathogen

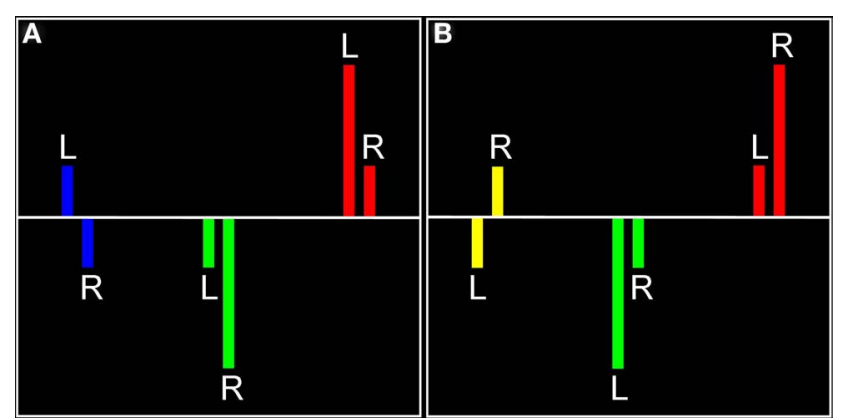

FIGURE 1 | Visual representation of laterality effects. For each significant effect in each hemisphere (A, left hemisphere; $\mathbf{B}$, right hemisphere), there are three possible relationships, each indicated by a different color. Red represents regions in which there was positive signal change in both hemispheres, with the indicated hemisphere having a more positive signal change (found in left or right hemisphere). Blue represents positive signal change in the left hemisphere and negative signal change in the right hemisphere (found in left hemisphere only). Yellow represents negative signal change in the left hemisphere and positive signal change in the right hemisphere (found in right hemisphere only). Green represents negative signal change in both hemispheres, with the indicated hemisphere having a smaller negative signal change (there were no significant voxels for this relationship) 
Table 2 | Areas of engagement in the left hemisphere in three studies of moral violation processing.

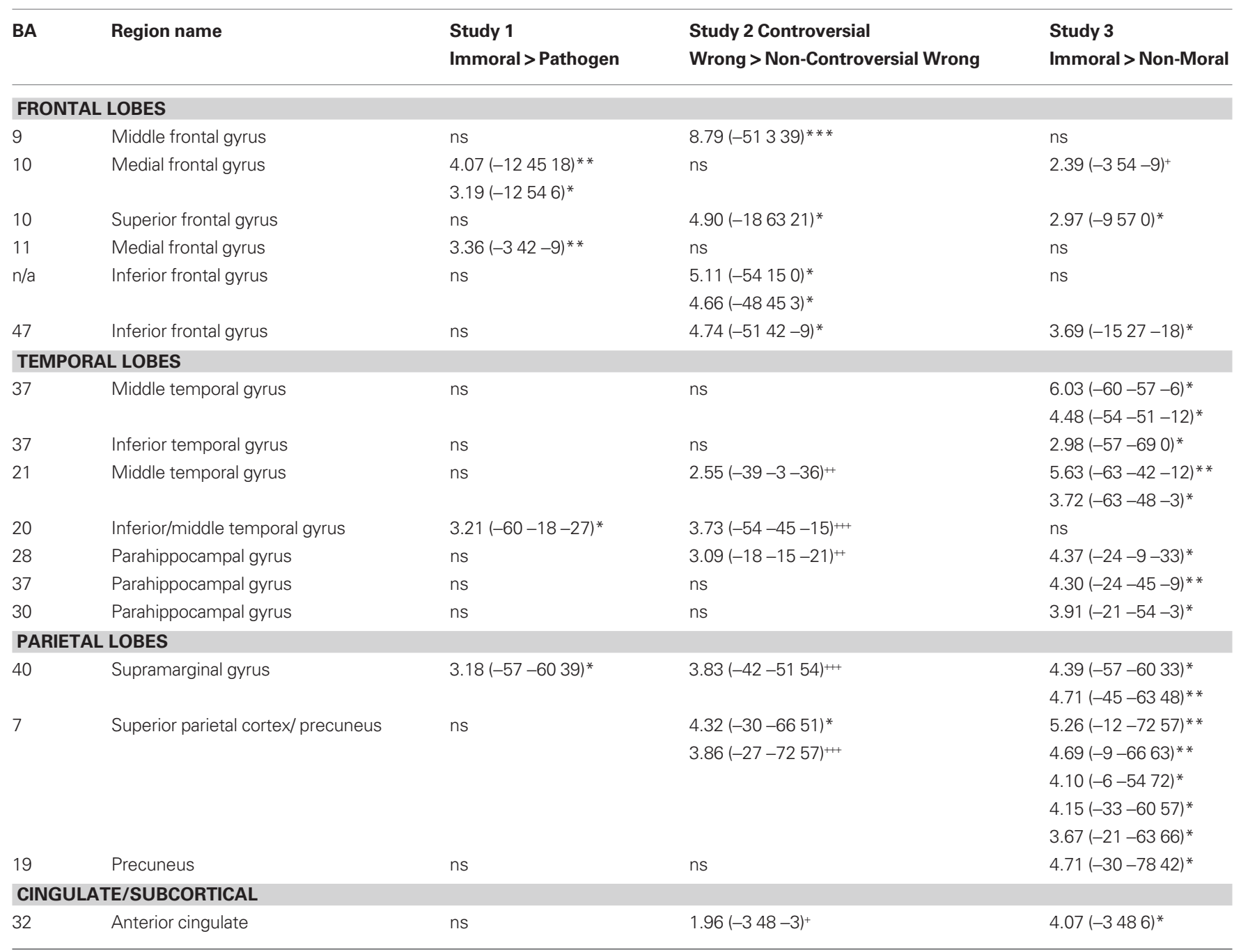

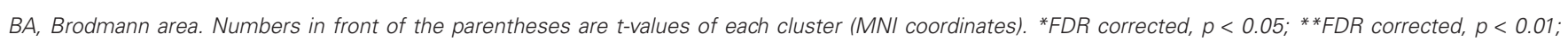
${ }^{*}{ }^{*} F D R$ corrected, $p<0.001$; Uncorrected statistics are also reported for completeness. ${ }^{+}$Uncorrected, $p<0.05 .{ }^{++}$Uncorrected, $p<0.01$. ${ }^{++}$Uncorrected, $p<0.001$.

can be found in Figure 2. The components of the Immoral $>$ Neutral contrast were analyzed separately (Figure 5) to ensure that the effects were not due solely to the sexual nature of the stimuli.

\section{Laterality analysis of Immoral > Neutral}

A laterality analysis was performed to identify which regions were preferentially engaged during the presentation of negative morally laden (incest and non-sexual immoral) stimuli compared with neutral stimuli (Figure 3). This analysis (false discovery rate, FDR corrected, $p<0.001)$ revealed significant engagement of the left superior frontal (Brodmann area, BA 8) and medial frontal gyri (BA 8/10), left inferior temporal/fusiform gyrus (BA 37), left supramarginal gyrus (BA 40), left posterior cingulate/precuneus (BA 31/7/24), left thalamus, right precentral gyrus (BA 6), and right cerebellum.

\section{Laterality analysis of Immoral > Pathogen}

This laterality analysis was performed to identify which regions were preferentially engaged during the processing of negative morally laden stimuli compared with disgusting, non-moral stimuli (Figure 4; Table 2). This analysis (FDR corrected, $p<0.05)$ revealed significant engagement of the left medial frontal gyri (BA 10/11), right medial frontal gyrus (BA 32), left inferior temporal gyrus (BA 20 ), right middle (BA 21) and superior (BA 22) temporal gyri, right inferior parietal lobe (BA 40), and right cingulate gyrus (BA 31 ).

\section{STUDY 2}

The main effects of judging non-controversial wrong, not wrong, and controversial acts have been described elsewhere, and the main effects of Controversial Wrong > Non-Controversial Wrong can be found in Figure 2.

\section{Laterality analysis of Controversial Wrong > Non-Controversial Wrong}

Brain activity associated with judging controversial statements to be wrong was compared to brain activity associated with judging non-controversial statements to be wrong (Figure 6; Table 2). Laterality analysis (FDR corrected, $p<0.05$ ) revealed significant engagement in left middle (BA 9) and superior (BA 10) frontal gyri, left inferior frontal gyrus (BA 47), and left superior parietal cortex (BA 7). 


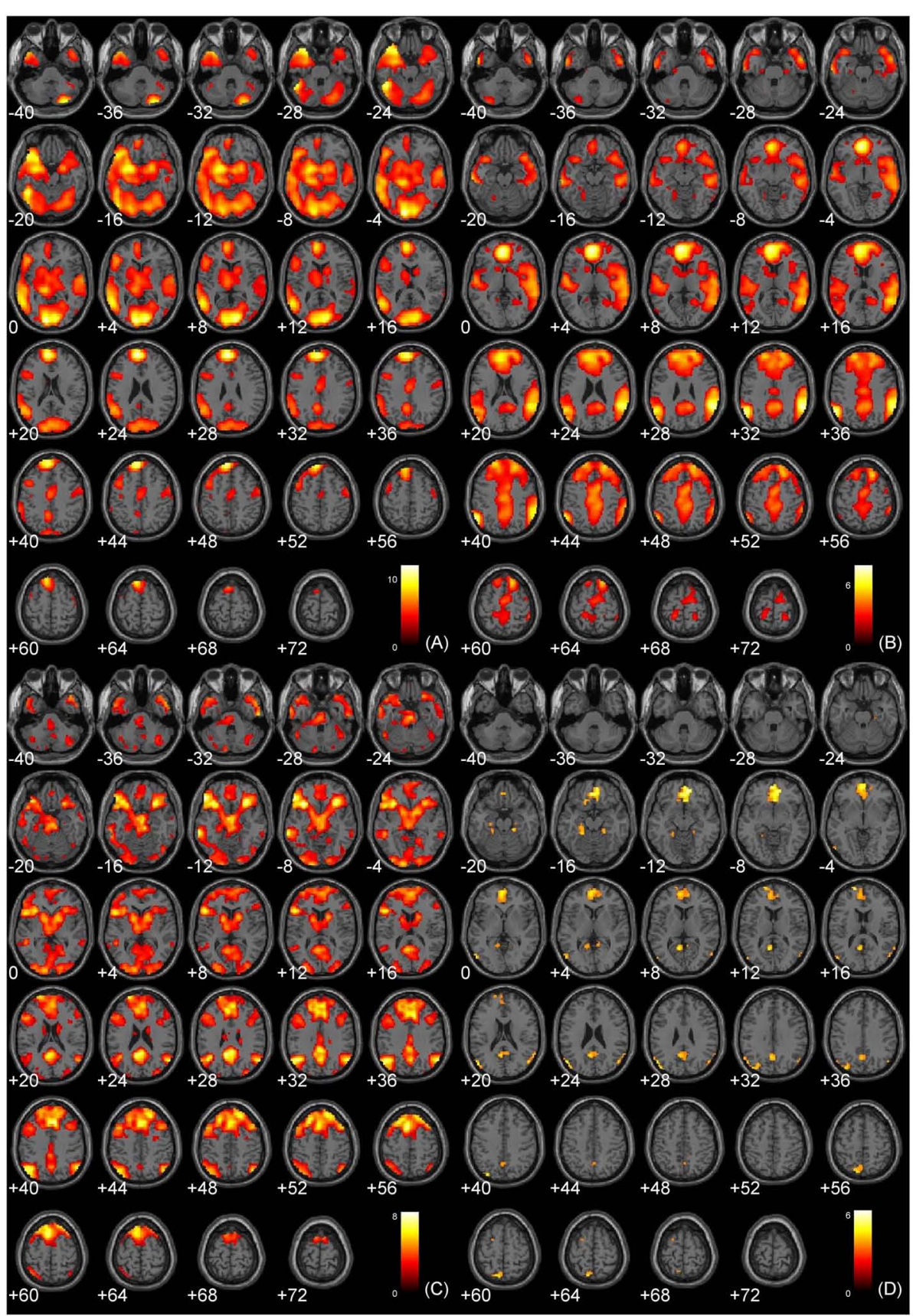

FIGURE 2 | Main effects analyses. (A) Study 1: Immoral > Neutral (FDR corrected, $p<0.001$ ); (B) Study 1: Immoral $>$ Pathogen (FDR corrected, $p<0.05$ ); (C) Study 2: Controversial Wrong > Non-Controversial Wrong (FDR corrected, $p<0.05$ ); (D) Study 3: Immoral $>$ Non-Moral (FDR corrected, $p<0.05$ ).

\section{STUDY 3}

The main effects of immoral vs. non-moral picture viewing have been described elsewhere (Harenski et al., 2008), and can also be found in Figure 2.

\section{Laterality analysis of Immoral > Non-Moral}

Brain activity associated with viewing pictures depicting immoral content was compared with brain activity associated with pictures without moral content (Figure 7; Table 2). Laterality analysis (FDR corrected, $p<0.05)$ revealed significant engagement of the left middle temporal and fusiform gyri (BA 37), left superior parietal/ precuneus (BA 7/19), left supramarginal gyrus (BA 40), left medial prefrontal cortex extending into anterior cingulate (BA 10/32), and left parahippocampal gyrus (BA 28/36/19).

\section{Laterality analysis of Non-Moral> Neutral}

A laterality analysis of brain activity associated with pictures without moral content compared to a baseline condition was carried out to evaluate the possibility that all stimuli in this study preferentially engaged the left hemisphere (FDR corrected, $p<0.05$ ). This revealed 


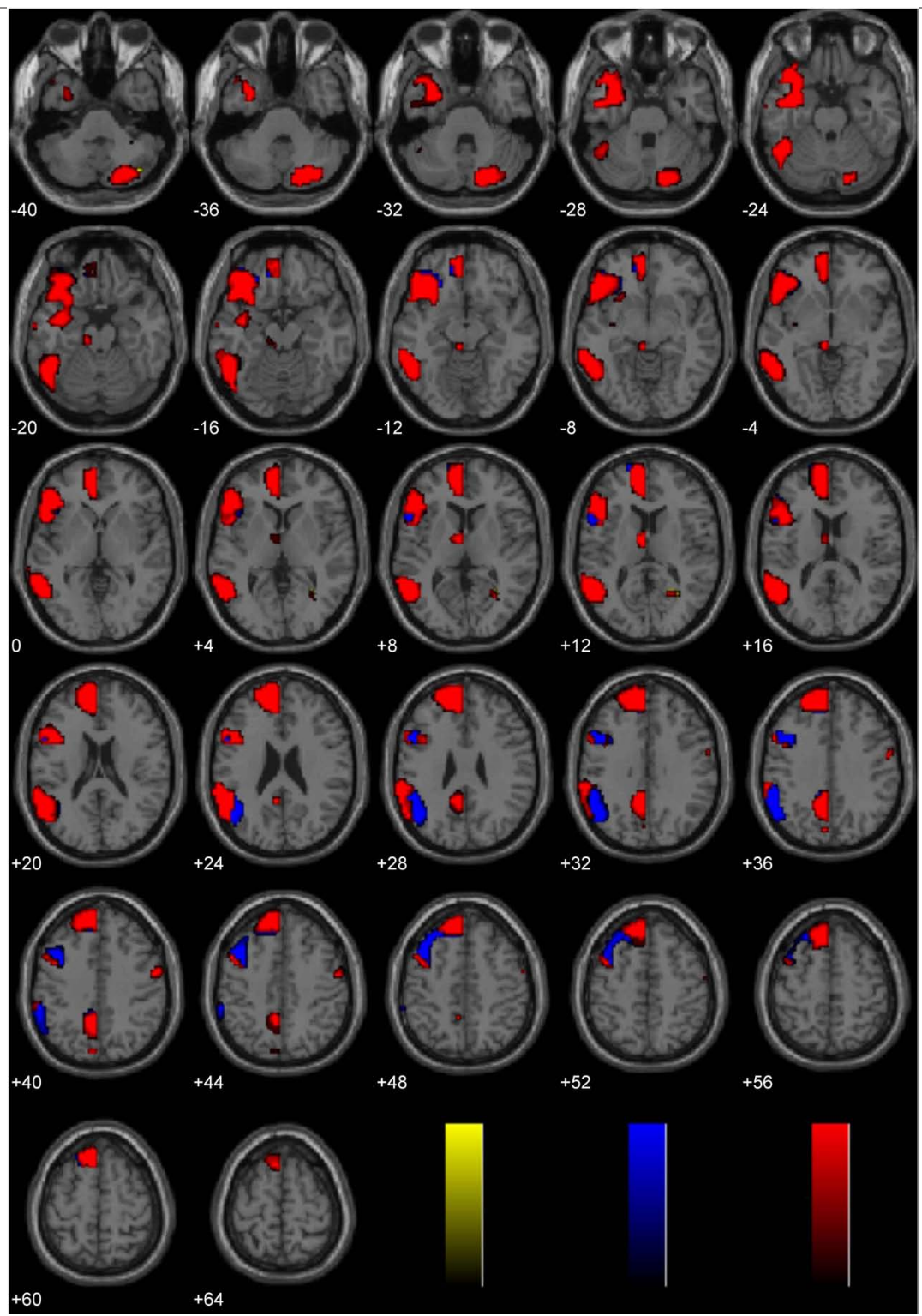

FIGURE 3 | Study 1: Immoral > Neutral. Depicted here are areas in which there is a significant difference in the amplitude of the hemodynamic response between hemispheres, limited to areas where there was also a significant main effect (results are overlaid on SPM5 canonicalT1 image, FDR corrected, $p<0.001)$. Areas of significant engagement include the left superior frontal and medial frontal gyri, left middle, and inferior temporal/fusiform gyrus, left supramarginal gyrus, left posterior cingulate/precuneus, left thalamus, right precentral gyrus, and right cerebellum. significant engagement in right-sided regions only, and included middle temporal gyrus (BA 37), lingual and fusiform gyri (BA 18), calcarine sulcus/cuneus (BA 17), and postcentral gyrus (BA 2). These regions have not been included in typical "morality networks."

\section{LATERALIZATION EFFECTS COMMON TO PROCESSING DIVERSE MORAL STIMULI}

The three main studies utilized in the present laterality analyses (Immoral $>$ Pathogen, Controversial Wrong $>$ Non-Controversial Wrong, Immoral > Non-Moral) employed diverse morally laden stimuli. Despite this assortment, a number of regions were found to be common areas of engagement during the processing of negative morally laden stimuli (Figure 8). Due to the stringency of a conjunction analysis of three distinct studies, results are presented at uncorrected levels. Commons areas of engagement in the left hemisphere were found in medial frontal gyrus (BA 10), superior frontal gyrus (BA 8), posterior cingulate (BA 31), temporoparietal junction (BA 39), middle temporal gyrus (BA 21), and inferior temporal gyrus (BA 20). Regions in the right hemisphere were anterior cingulate (BA 32) and temporoparietal junction (BA 39).

\section{DISCUSSION}

This study was designed to investigate the functional hemispheric laterality of processing immoral acts. Across three distinct paradigms, we found that processing moral violations more strongly 


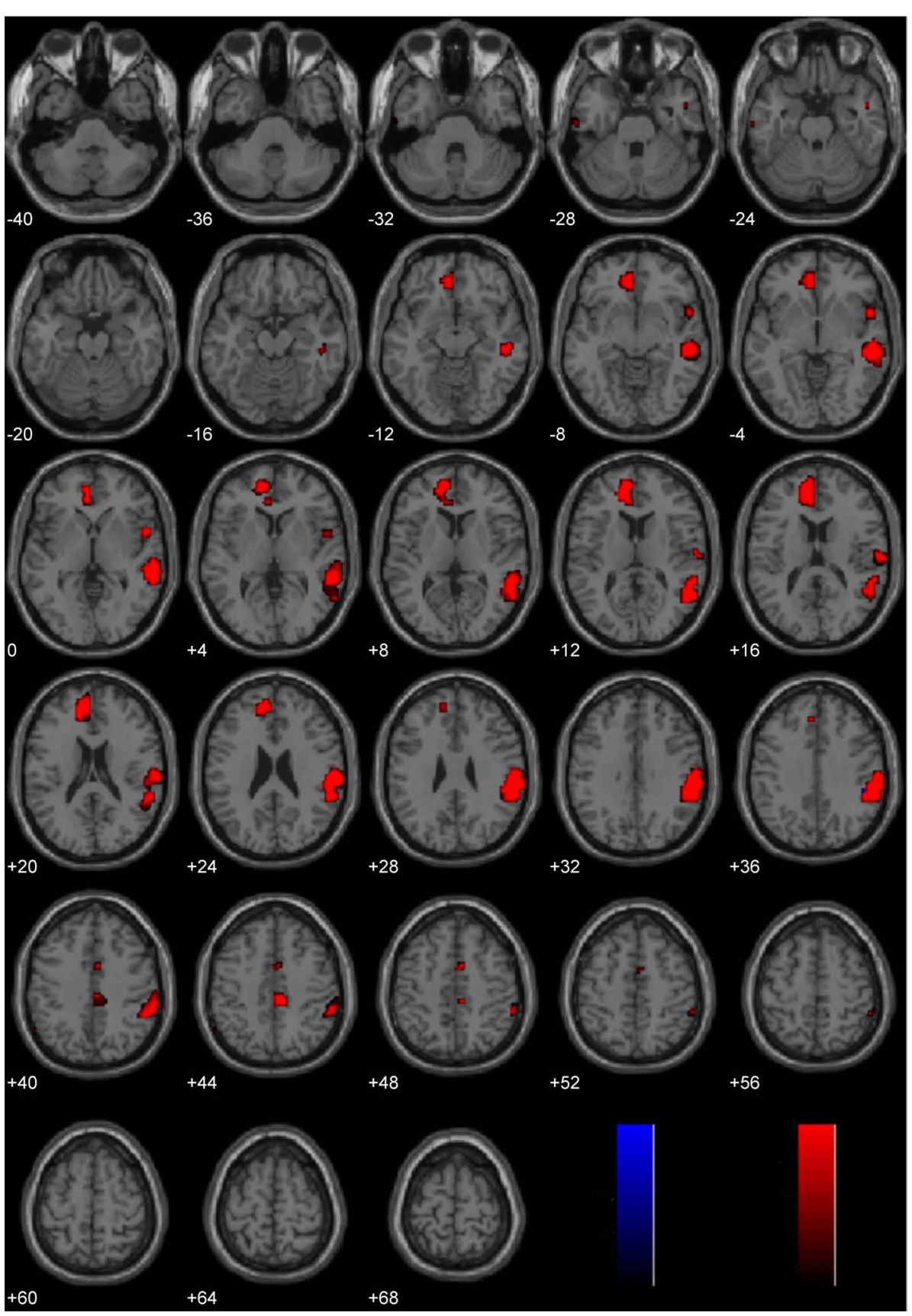

FIGURE 4 | Study 1: Immoral > Pathogen. Depicted here are areas in which there is a significant difference in the amplitude of the hemodynamic response between hemispheres, limited to areas where there was also a significant main effect (results are overlaid on SPM5 canonicalT1 image,
FDR corrected, $p<0.05)$. Areas of significant engagement include the left medial frontal gyri, right medial frontal gyrus, left inferior temporal gyrus, right middle and superior temporal gyri, right inferior parietal lobe, and right cingulate gyrus. engages the left hemisphere than the right hemisphere. Specifically, the left greater than right hemisphere asymmetry was found for processing negative morally laden stimuli in the linguistic and pictorial domain, during tasks that focused either on the implicit or explicit processing of moral content, and for different classes of stimuli (sexual and non-sexual). These results highlight the consistency of left greater than right hemisphere asymmetry for processing diverse forms of immoral stimuli. Importantly, this consistency cannot be attributed to a generalized left hemisphere bias for the stimuli in these three studies, because the NonMoral $>$ Neutral contrast in Study 3 was right-lateralized, and the Immoral $>$ Pathogen contrast in Study 1 contained substantial clusters in the right hemisphere. Additionally, valence or emotional arousal cannot explain the results, because the immoral and non-moral stimuli in Study 3 were equated for arousal and valence, and the controversial and non-controversial stimuli in 


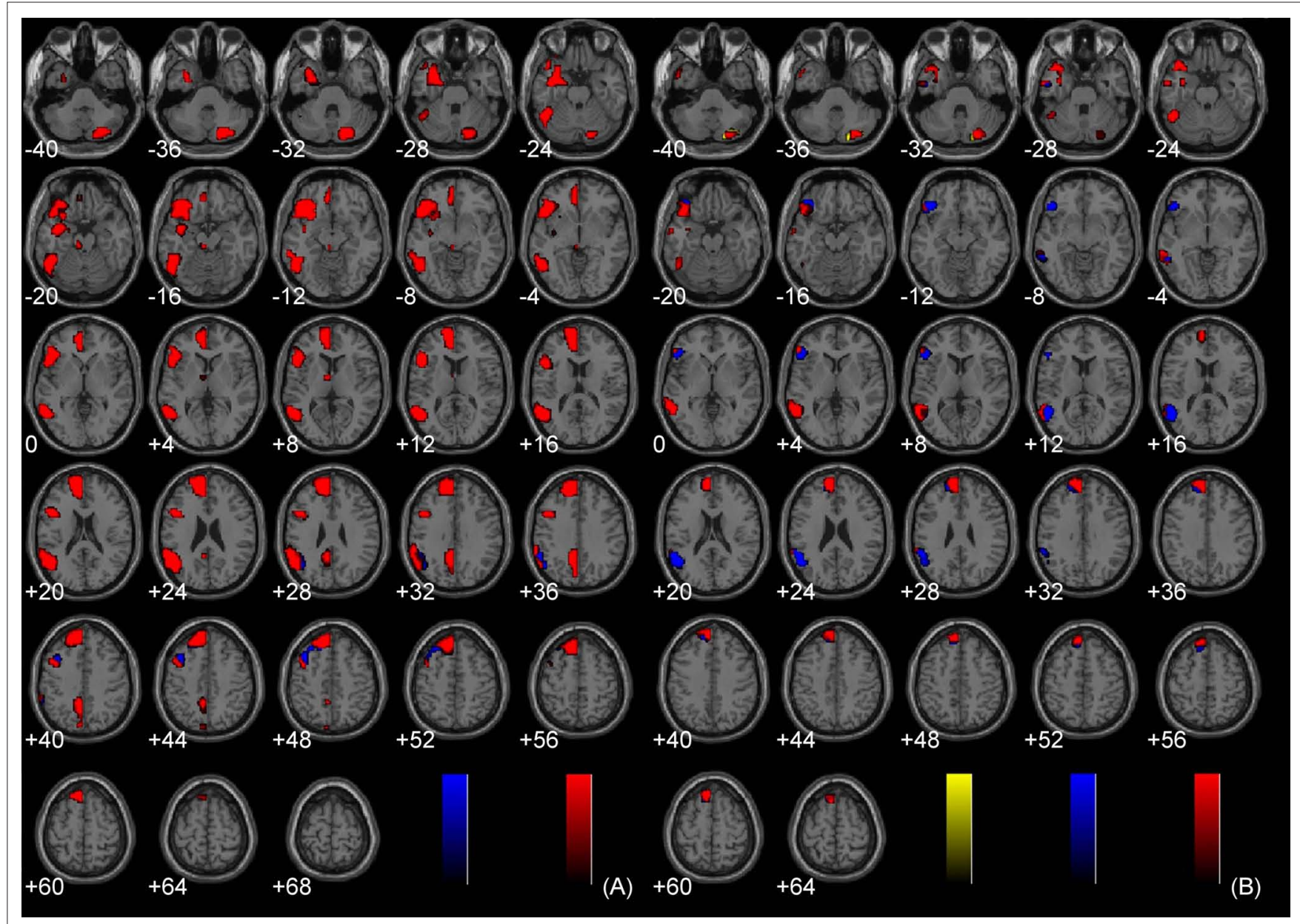

FIGURE 5 | Components of Immoral $>$ Neutral. (A) Incest $>$ Neutral (FDR corrected, $p<0.001$ ); (B) Non-Sexual Immoral $>$ Neutral (FDR corrected, $p<0.001$ ).

Study 2 and the immoral and pathogen stimuli in Study 1 were both negatively valenced, yet these comparisons still indicated a left hemisphere bias.

The extant literature supports at least two plausible interpretations for the left greater than right asymmetry for processing immoral stimuli. One interpretation is that morally relevant negative emotions are "social emotions" that, unlike other negative emotions, are lateralized to the left hemisphere. This interpretation is supported by Ross et al. (1994), but contradicts both the valence hypothesis (i.e., the left hemisphere processes positive emotions whereas the right hemisphere processes negative emotions) and the right hemisphere hypothesis (which states that emotion processing is right-lateralized).

An alternative interpretation is that negative emotions are rightlateralized, but moral judgments are left-lateralized. Perhaps negative emotions on the right side draw our attention to a moral issue and thereby lead to moral cognition (which may include other emotions) and reasoning on the left side. It is important to note that in Studies 2 and 3, participants assessed moral content in both conditions (Controversial Wrong $>$ Non-Controversial Wrong and Immoral > Non-Moral), so it is likely not the evaluation of stimuli for moral content per se that engages the left hemisphere. Rather, it may be the judgment that something is a moral violation. Thus, the social emotion perspective may be the most parsimonious interpretation of the current results.

The present data, while suggestive, do not unequivocally indicate that the left hemisphere is more "necessary" for processing moral violations than the right hemisphere. Processing of moral stimuli engaged several right hemisphere regions, but the present analyses suggest that the left hemisphere is showing greater engagement, reflected as higher amplitude hemodynamic response, than the right hemisphere. This asymmetric left hemisphere engagement may be a reflection of greater computational processing or some other cognitive process(es) such as categorical rather than graded judgment (e.g., Kosslyn et al., 1989). Right hemisphere involvement has been found in other studies of moral processing. Recent studies of callosotomy patients (Miller et al., 2010) and transcranial magnetic stimulation (Young et al., 2010) have implicated the right temporoparietal junction as being crucial for belief attribution, while others (Young et al., 2007; Young and Saxe, 2008, 2009), have likewise found strong evidence for the right temporoparietal junction in moral processing. There is evidence to suggest that moral judgment is not a unitary concept (Haidt, 2001; Greene et al., 2004; Cushman et al., 2006), so it is possible that whereas 


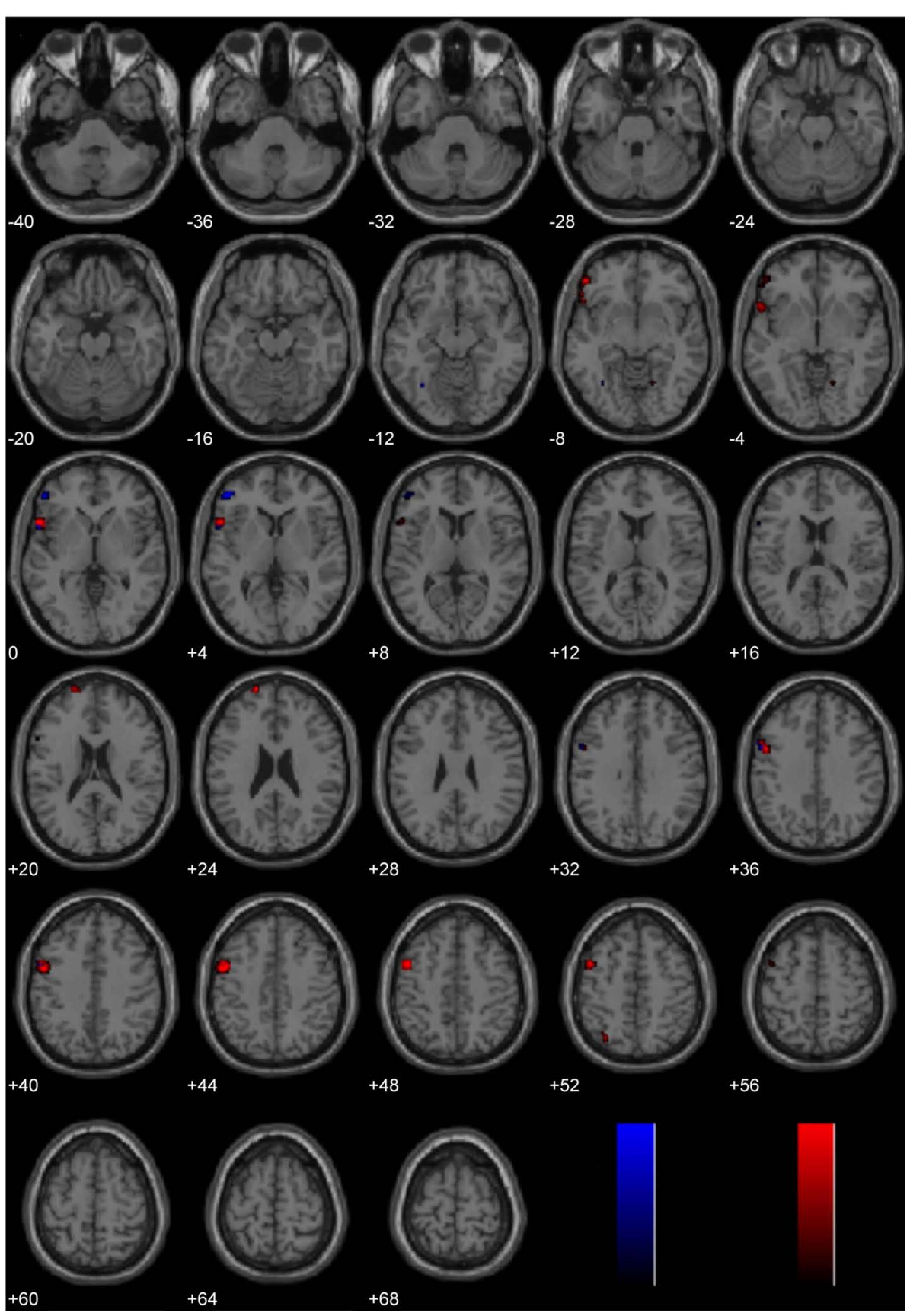

FIGURE 6 | Study 2: ControversialWrong > Non-Controversial Wrong.

Depicted here are areas in which there is a significant difference in the amplitude of the hemodynamic response between hemispheres, limited to areas where there was also a significant main effect (results are overlaid on SPM5 canonicalT1 image, FDR corrected, $p<0.05)$. Areas of significant engagement include left middle and superior frontal gyri, left inferior frontal gyrus, and left superior parietal cortex. belief attribution relies on the right temporoparietal junction, moral violation processing relies more heavily on the left hemisphere than the right. Additional studies of patients with unilateral brain damage, commisurotomy, or similar would help more fully delineate necessary from sufficient brain regions associated with processing negative morally laden stimuli. Future studies should also evaluate the role of gender in the hemispheric lateralization of moral processing.
Interestingly, the observed pattern of neural engagement common to all three studies (Figure 8) is remarkably similar to the default mode network. This network of ventral medial prefrontal cortex, posterior cingulate, inferior parietal lobule, lateral temporal cortex, dorsal medial prefrontal cortex, and hippocampus, has been described as an organized mode of brain function that is suspended during goal-directed behaviors (Raichle et al., 2001; Buckner et al., 2008). It is also thought to be important in mentalization and 


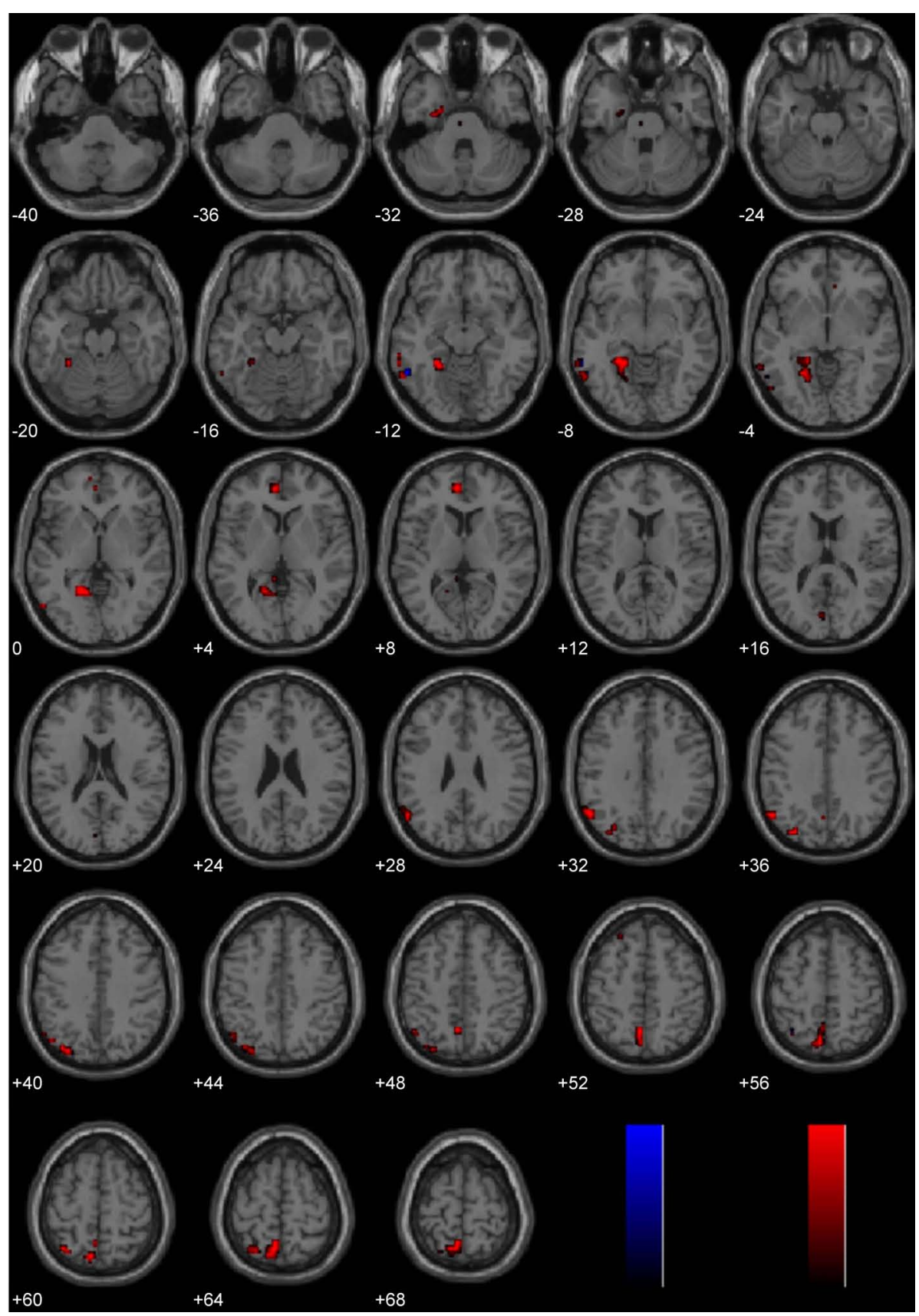

FIGURE 7 | Study 3: Immoral > Non-Moral. Depicted here are areas in which there is a significant difference in the amplitude of the hemodynamic response between hemispheres, limited to areas where there was also a significant main effect (results are overlaid on SPM5 canonicalT1 image
FDR corrected, $p<0.05)$. Areas of significant engagement include the left middle temporal gyrus, left fusiform gyrus, left supramarginal gyrus, left superior parietal/precuneus, bilateral anterior cingulate, and left parahippocampal gyrus. introspection, which are related to theory of mind (Gallagher and Frith, 2003). As theory of mind is an important aspect of moral judgment (Young et al., 2007), future studies should examine the lateralization of theory of mind processing and how this might be represented during moral judgment.

Why might the processing of moral violations be lateralized? It is well-established that hemispheric lateralization can be an efficient strategy for motor, perceptual, and cognitive functions
(Levy, 1977; Vallortigara and Rogers, 2005), avoiding the duplication of bilateral processes. Hemispheric specialization has been observed in non-humans as well, suggesting a shared evolved capacity emerging as early as hundreds of millions of years ago (Vallortigara et al., 1999). It may be that the lateralization of moral violation processing, like other lateralized higher-order functions, arose early in our evolution to increase neural capacity and efficiency. 


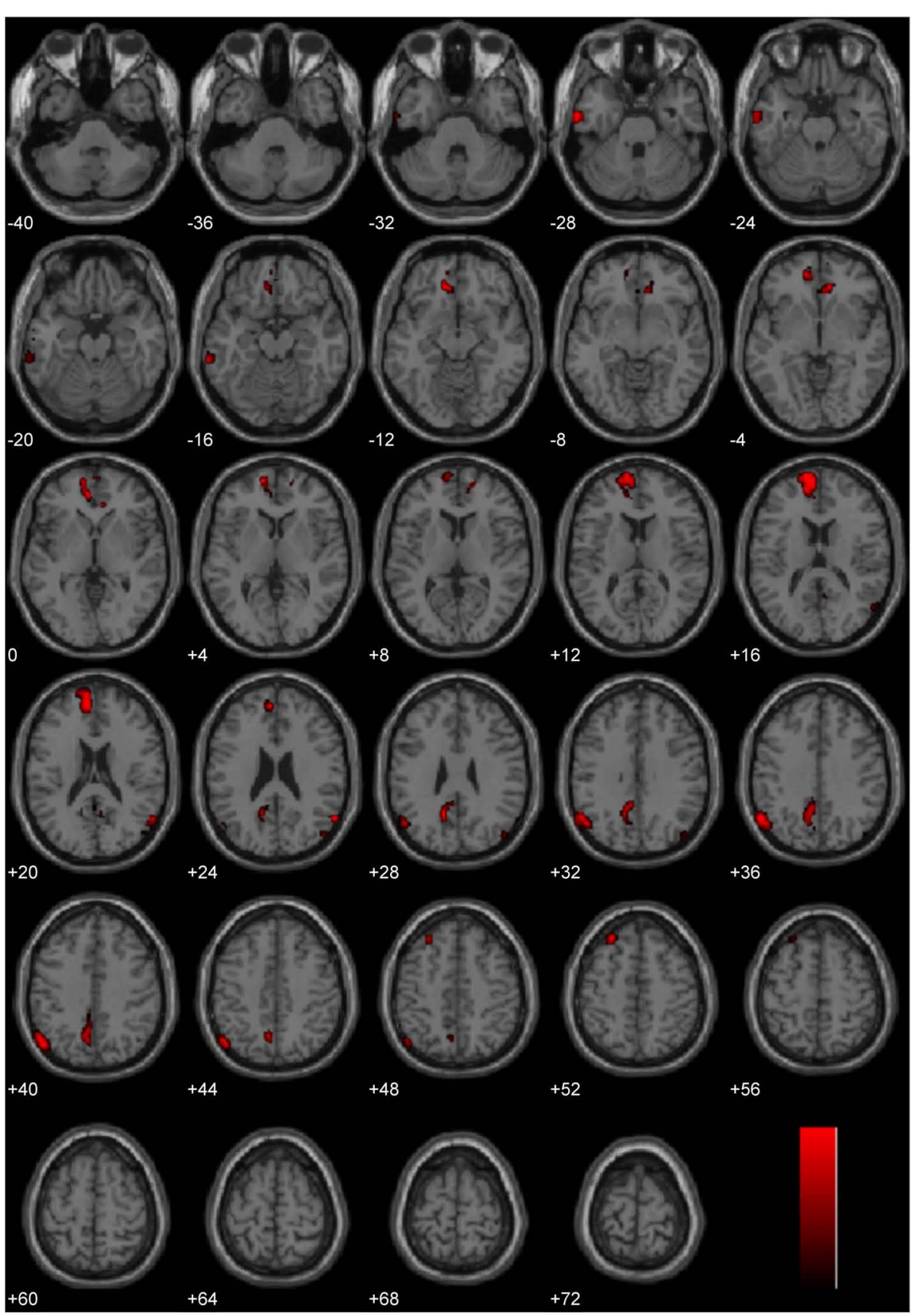

FIGURE 8 | Conjunction analysis. Depicted here are areas of significant engagement that are common to all three studies (Study 1: Immoral > Pathogen, Study 2: Controversial Wrong > Non-Controversial Wrong, and Study 3: Immoral > Non-Moral). Results are overlaid on SPM5 canonical T1 image, uncorrected, $p<0.005$. Significant areas of engagement include the left medial frontal gyrus, left posterior cingulate, left temporoparietal junction, right anterior cingulate, and right temporoparietal junction.
Our goal here was to contribute to the characterization of evolved psychological adaptations by describing the neural organization of moral processing. Specialized neural circuitry has also been identified for a number of proposed adaptations including social exchange (Stone et al., 2002; Ermer et al., 2006), facial recognition (Grill-Spector et al., 2004), and theory of mind (Baron-Cohen et al., 1999). Future studies investigating morality should address the computational nature of moral processing, not just its functional asymmetry as we provide here. We hope that our findings serve as a starting point and inform future functionalist hypotheses.

In summary, these results indicate that there is a left hemisphere bias for the processing of immoral stimuli across multiple domains. This effect was demonstrated in three distinct paradigms, using 
both pictures and phrases. This work suggests that complex social cognitive processes may have evolved to utilize specific, left-lateralized cognitive circuits, and that processing immoral acts shows functional specialization akin to other perceptual, linguistic, and cognitive abilities.

\section{REFERENCES}

Baron-Cohen, S., Ring,H.A., Wheelwright, S., Bullmore, E. T., Brammer, M. J., Simmons, A., and Williams, S. C. R. (1999). Social intelligence in the normal and autistic brain: an fMRI study. Eur. J. Neurosci. 11, 1891-1898.

Borod, J. C., Haywood, C. S., and Koff, E. (1997). Neuropsychological aspects of facial asymmetry during emotional expression: a review of the normal adult literature. Neuropsychol. Rev. 7, 41-60.

Braun, M., Traue, H. C., Frisch, S., Deighton, R.M., and Kessler,H. (2005). Emotion recognition in stroke patients with left and right hemispheric lesion: results with a new instrument - the FEEL test. Brain Cogn. 58, 193-201.

Broca, P. (1887). Rapport sur un memoire de M. Armand de Fleury intitulé: de l'inegalité dynamique des deux hemisphères cerébraux. Bull. Acad. Natl. Med. 6, 508-539.

Buckner, R. L., Andrews-Hanna, J. R., and Schacter, D. L. (2008). The brain's default network: anatomy, function, and relevance to disease. $\operatorname{Cog} n$. Neurosci. 1124, 1-38.

Canli, T., Desmond, J. E., Zhao, Z., Glover, G., and Gabrieli, J. D. E. (1998). Hemispheric asymmetry for emotional stimuli detected with fMRI. Neuroreport 9, 3233-3239.

Cushman, F., Young, L., and Hauser, M. (2006). The role of conscious reasoning and intuition in moral judgment: testing three principles of harm. Psychol. Sci. 17, 1082-1089.

Davidson, R. J. (1993). Parsing affective space: perspectives from neuropsychology and psychophysiology. Neuropsychology 7, 464-475.

Davidson, R. J. (1998). Affective style and affective disorders: perspectives from affective neuroscience. Cogn. Emot. 12, 307-330.

Demaree, H. A., Everhart, D. E., Youngstrom, E. A., and Harrison, D. W. (2005). Brain lateralization of emotional processing: historical roots and a future incorporating "dominance." Behav. Cogn. Neurosci. Rev. 4, 3-20.

Ermer, E., Guerin, S., Cosmides, L., Tooby, J., and Miller, M. (2006). Theory of mind broad and narrow: reasoning about social exchange engages ToM areas, precautionary reasoning does not. Soc. Neurosci. 1, 196-219.
Freire, L., Roche, A., and Mangin, J. F. (2002). What is the best similarity measure for motion correction in fMRI time series? IEEE Trans. Med. Imaging 21, 470-484.

Gainotti, G. (1997). "Emotional disorders in relation to unilateral brain damage," in Behavioral Neurology and Neuropsychology, eds T. E. Feinberg and M. Farah (New York: McGrawHill), 691-698.

Gainotti, G. (2000). "Neuropsychological theories of emotions," in The Neuropsychology of Emotions, ed. J. Borod (New York: Oxford University Press), 214-238.

Gallagher, H. L., and Frith, C. D. (2003). Functional imaging of "theory of mind." Trends Cogn. Sci. 7, 77-83.

Greene, J., and Haidt, J. (2002). How (and where) does moral judgment work? Trends Cogn. Sci. 6, 517-523.

Greene, J. D., Nystrom, L. E., Engell, A. D., Darley, J. M., and Cohen, J. D. (2004). The neural bases of cognitive conflict and control in moral judgment. Neuron 44, 389-400.

Grill-Spector, K., Knouf, N., and Kanwisher, N. (2004). The fusiform face area subserves face perception, not generic within-category identification. Nat. Neurosci. 7, 555-562.

Haidt, J. (2001). The emotional dog and its rational tail: a social intuitionist approach to moral judgment. Psychol. Rev. 108, 814-834.

Harenski, C. L., Antonenko, O., Shane, M. S., and Kiehl, K.A. (2008). Gender differences in neural mechanisms underlying moral sensitivity. Soc. Cogn. Affect. Neurosci. 3, 313-321.

Harenski, C. L., and Hamann, S. (2006). Neural correlates of regulating negative emotions related to moral violations. Neuroimage 30, 313-324.

Kiehl, K. A., Stevens, M. C., Laurens, K. R., Pearlson, G., Calhoun, V. D., and Liddle, P. F. (2005). An adaptive reflexive processing model of neurocognitive function: supporting evidence from a large scale $(n=100)$ MRI study of an auditory oddball task. Neuroimage 25, 899-915.

Kosslyn, S. M., Koenig, O., Barrett, A., Cave, C. B., Tang, J., and Gabrieli, J. D. (1989). Evidence for two types of spatial representations: hemispheric specialization for categorical and coordinate relations. J. Exp.

\section{ACKNOWLEDGMENTS}

The authors wish to thank Keith Harenski and Kevin Bache for their assistance with the preparation of the manuscript. This research was supported by the National Institutes of Health, under grant R01 MH 070539 (to Dr. Kent A. Kiehl).

Psychol. Hum. Percept. Perform. 15, 723-735.

Kurzban, R., Dukes, A., and Weeden, J. (2010). Sex, drugs and moral goals: reproductive strategies and views about recreational drugs. Proc. R. Soc. B 277, 3501-3508.

Lang, P. J., Bradley, M. M., and Cuthbert, B. N. (1995). International Affective Picture System (IAPS): Technical Manual and Affective ratings (Tech. Rep. No. A-4). Gainesville, FL: University of Florida, Center for Research in Psychophysiology.

Levy, J. (1977). The mammalian brain and the adaptive advantage of cerebral asymmetry. Ann. N.Y. Acad. Sci. 299, 264-272.

Lieberman, D., Tooby, J., and Cosmides, L. (2003). Does morality have a biological basis? An empirical test of the factors governing moral sentiments relating to incest. Proc. R. Soc. Lond., B, Biol. Sci. 270, 819-826.

Meadows, M. E., and Kaplan, R. F. (1994). Dissociation of autonomic and subjective responses to emotional slides in right-hemisphere damaged patients. Neuropsychologia 32, 847-856.

Miller, M. B., Sinnott-Armstrong, W., Young, L., King, D., Paggi, A., Fabri, M., and Gazzaniga, M. S. (2010). Abnormal moral reasoning in complete and partial callosotomy patients. Neuropsychologia 48, 2215-2220.

Moll, J., Zahn, R., de Oliveira-Souza, R., Krueger, F., and Grafman, J. (2005). The neural basis of human moral cognition. Nat. Rev. Neurosci. 6, 799-809.

Nichols, T. E., Brett, M., Andersson, J. Wager, T., and Poline, J. B. (2005). Valid conjunction inference with the minimum statistic. Neuroimage 25 , 653-660.

Raichle, M. E., MacLeod,A. M., Snyder, A Z., Powers, W. J., Gusnard, D. A., and Shulman, G. L. (2001). A default mode of brain function. Proc. Natl. Acad. Sci. U.S.A. 98, 676-682.

Ross, E. D. (1984). Right hemisphere's role in language, affective behavior and emotion. Trends Neurosci. 7, 342-346.

Ross, E. D., Homan, R. W., and Buck, R. (1994). Differential hemispheric lateralization of primary and social emotions - implications for develop- ing a comprehensive neurology for emotions, repression, and the subconscious. Neuropsychiatry Neuropsychol. Behav. Neurol. 7, 1-19.

Schaich Borg, J., Lieberman, D., and Kiehl, K. A. (2008). Infection, incest, and iniquity: investigating the neural correlates of disgust and morality. J. Cogn. Neurosci. 20, 1529-1546.

Stevens, M. C., Calhoun, V. D., and Kiehl, K. A. (2005). Hemispheric differences in hemodynamics elicited by auditory oddball stimuli. Neuroimage 26, 782-792.

Stone, V., Cosmides, L., Tooby, J., Kroll, N., and Knight, R. (2002). Selective impairment of reasoning about social exchange in a patient with bilateral limbic system damage. Proc. Natl. Acad. Sci. U.S.A. 99, 11531-11536.

Tamietto, M., Adenzato, M., Geminiani, G., and de Gelder, B. (2007). Fast recognition of social emotions takes the whole brain: interhemispheric cooperation in the absence of cerebral asymmetry. Neuropsychologia 45, 836-843.

Tangney, J. P., Stuewig, J., and Mashek, D. J. (2007). Moral emotions and moral behavior. Annu. Rev. Psychol. 58, 345-372.

Vallortigara, G., and Rogers, L. J. (2005). Survival with an asymmetrical brain: advantages and disadvantages of cerebral lateralization. Behav. Brain Sci. 28, 575-633.

Vallortigara, G., Rogers, L. J., and Bisazza, A. (1999). Possible evolutionary origins of cognitive brain lateralization. Brain Res. Rev. 30, 164-175.

Wittling, W. (1995). "Brain asymmetry in the control of autonomic-physiologic activity," in Brain Asymmetry, eds R. J. Davidson and K. Hugdahl (Cambridge, MA: MIT Press), 305-357.

Wittling, W., and Roschmann, R. (1993). Emotion-related hemisphere asymmetry: subjective emotional responses to laterally presented films. Cortex 29 , 431-448.

Young, L., Camprodon, J. A., Hauser, M., Pascual-Leone, A., and Saxe, R. (2010). Disruption of the right temporoparietal junction with transcranial magnetic stimulation reduces the role of beliefs in moral judgments. Proc. Natl. Acad. Sci. U.S.A. 107, 6753-6758. 
Young, L., Cushman, F., Hauser, M., and Saxe, R. (2007). The neural basis of the interaction between theory of mind and moral judgment. Proc. Natl. Acad. Sci. 104, 8235-8240.

Young, L., and Koenigs, M. (2007). Investigating emotion in moral cognition: a review of evidence from functional neuroimaging and neuropsychology. Br. Med. Bull. 84, 69-79.

Young, L., and Saxe, R. (2008). The neural basis of belief encoding and integration in moral judgment. Neuroimage 40, 1912-1920.

Young, L., and Saxe, R. (2009). Innocent intentions: a correlation between forgiveness for accidental harm and neural activity. Neuropsychologia 47, 2065-2072.

Conflict of Interest Statement: The authors declare that the research was conducted in the absence of any commercial or financial relationships that could be construed as a potential conflict of interest.

Received: 05 April 2010; paper pending published: 07 September 2010; accepted: 07 December 2010; published online: 30 December 2010.

Citation: Cope LM, Schaich Borg J, Harenski CL, Sinnott-Armstrong W, Lieberman D, Nyalakanti PK, Calhoun VD and Kiehl KA (2010) Hemispheric asymmetries during processing of immoral stimuli. Front. Evol. Neurosci. 2:110. doi $10.3389 /$ fnevo. 2010.00110

Copyright (c) 2010 Cope, Schaich Borg, Harenski, Sinnott-Armstrong, Lieberman, Nyalakanti, Calhoun and Kiehl. This is an open-access article subject to an exclusive license agreement between the authors and the Frontiers Research Foundation, which permits unrestricted use, distribution, and reproduction in any medium, provided the original authors and source are credited. 